\author{
Popenaka $\mathrm{A}^{1}$., Kolbasin $\mathrm{O}^{2}$., Maslova $\mathrm{N}^{2}$. \\ ${ }^{1}$ Research and Production Enterprise TUNIKA Ltd, Kharkov, Ukraine \\ ${ }^{2} \mathrm{NSC}$ «Institute of metrology», Kharkov, Ukraine
}

\title{
EVALUATION OF THE MEASUREMENT UNCERTAINTY AT CALIBRATION OF DEVICES FOR ELECTRICAL ENERGY AND POWER METERS VERIFICATION
}

The technology of mode parameters vector measurement is described. Hardware-measuring-computer complex is suggested for testing a wide range of power measuring instruments. The dual channel synchronous analog-todigital converter $(A D C)$ connected to the voltage divider and the shunt is a single-phase power meter. Stages of calibration of electrical energy and power meters are considered. The measurement uncertainty at calibration of devices for electrical energy and power meters verification is evaluated. The results of the calibration of the two reference meters are presented as an example.

Key words: measurement uncertainty, vector measurement, calibration, power, electrical energy, AC voltage, electric current, analog-to-digital converter.

Nowadays cost of any production is determined by energy intensity of its manufacturing largely than by the material and even high technology.

Thus, special attention is paid to the accounting of energy and in particular electricity, which resulted in the rapid growth of production of electricity meters and improving their metrological characteristics.

This fact led to development of reference standards and metrological traceability to electrical power unit, which resulted in design of the appropriate hierarchical scheme [1].

At the same time, electric power counters began to be supplied with additional functions, such as the measurement of the electricity quality parameters.

In the past 10 years, it has initiated development of the complex of standards in the area of the electricity quality measuring, which is connected with the reference voltage and ampere standards, and existing independently of the traceability of electrical power unit.

This order of things has become habitual, and it has forgotten that the active power is the product of voltage, current and cosine of the angle between them.

On the other hand, in these latter years, vector measurement technology of mode parameters are implemented intensively in the energy systems of technically developed countries, especially voltage vector and current vector of the first harmonic.

Such measurements are performed with special devices, which are usually denoted by the abbreviation of PMU (Phasor Measurement Unit), or their functions are implemented in existing hardware, in particular the protection devices, recorders, and others.

These measurements, obtained at different points in the power system and synchronized in time, are the basis of the mode monitoring and create an information base for more effective solutions to many traditional and new challenges in the field of computer-aided dispatch and automatic management of emergency power systems [2].

Despite the fact that obtained voltage and current vectors allow us to evaluate power and energy, this method is not actively used for electricity metering.

The main reasons are insufficient accuracy of the obtaining phase vectors (currently accuracy at the level of tenth part of phase degree are considered acceptable [3]), and the lack of metrological support of such measurements.

However, there is the possibility of reference standards developing, which on the one hand provides electrical power unit, and the other hand applies the principles of vector measurements. Such standards are units of testing PMU devices of modern power system.

The imitator and meter of electrical power parameters IBПЕE-3Ф-1 was created. It was developed on the basis of hardware and software of measuring and computing set "Metrolog", which is based on vector voltage and current measurements and the frequency measurement.

The set is designed for calibration of a wide range of measuring instruments used in the power industry, as well as for laboratory and field measurements of electric quantities and their analysis.

The initial values for electric power unit realization in the set are the AC voltage and current units derived from the calibrator Fluke 5720A, which is based on thermal conversion. Voltage and current synchronization is carried out in the set.

In general, single-phase power meter is a dual channel synchronous analog-to-digital converter (ADC) connected to large-scale voltage and current converters, which are voltage divider and shunt.

Wherein divider and shunt should not only have a stable conversion factor, but does not make a phase shift. The production of such large-scale converters is a non-trivial task.

Applying the principle of vector measurements allowed to refuse such high requirements for large-scale converters as opportunity arose to independently calibrate the amplitude conversion factor and phase shift of "large-scale converter - ADC" system in the assembly.

Thus, instead of a precision nonreactive divider we apply two units: precision divider with unknown phase shift is used to scale the voltage amplitude, nonreactive divider with unstable division factor used to calibrate the voltage vector phase.

In order to obtain AC power unit, transducer composed of synchronous three-channel ADC was fabricated. 
The input $\mathrm{A}$ is connected to the precision voltage divider $\mathrm{PD}$, the input $\mathrm{B}$ is connected to the precision current shunt PSh, the input $\mathrm{C}$ is used as an auxiliary. Calibration algorithm of the system consists of three stages: $1)$.

Stage 1: Calibrating of the voltage channel (Figure

Nonreactive divider ND is connected to the input C. The required voltage $U_{\text {ref }}$ from the calibrator Fluke 5720A is supplied on precision and nonreactive dividers in parallel. The complex transfer characteristic of the channel $\mathrm{A}\left(\mathrm{K}_{\mathrm{A} 0}\right.$ and $\left.\psi_{\mathrm{A} 0}\right)$ is determined, and amplitude and phase voltage ratios are modified accordingly (1), (2) using the control program.

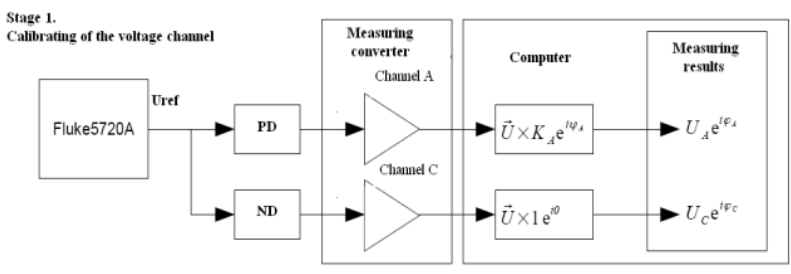

Figure 1. Calibrating of the voltage channel

$$
\begin{gathered}
K_{A}=\frac{K_{A O} \cdot \text { Uref }}{U_{A}} \\
\psi_{A}=\psi_{A O}+\varphi_{C}-\varphi_{A}
\end{gathered}
$$

Stage 2: Calibrating of the curent channel (Figure 2). Nonreactive shunt NSh is connected to the input C. The required current $\mathrm{I}_{\mathrm{ref}}$ from calibrator Fluke5720A is supplied on precision and nonreactive shunts, connected in series.

Complex transfer function $\left(\mathrm{K}_{\mathrm{B} 0}\right.$ and $\left.\psi_{\mathrm{B} 0}\right)$ of channel $\mathrm{B}$ is defined and the current amplitude and phase coefficients are modified accordingly (3), (4) using the control program.

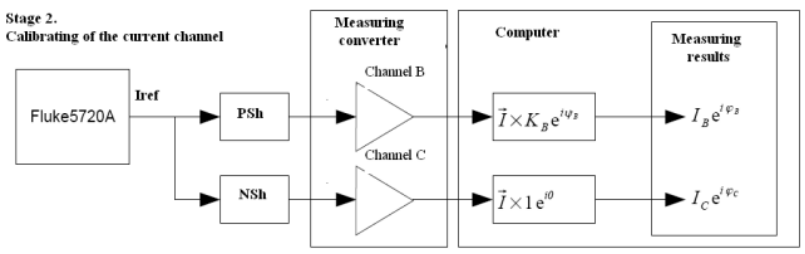

Figure 2. Calibrating of the current channel

$$
\begin{gathered}
K_{B}=\frac{K_{B 0} \cdot \text { Iref }}{I_{B}} \\
\psi_{B}=\psi_{B 0}+\varphi_{C}-\varphi_{B}
\end{gathered}
$$

Stage 3: Calibrating of the device under test (DUT) (Figure 3).

The input $\mathrm{C}$ is reset. The voltage from the test signals synthesizer is supplied to the PD and the device under test simultaneously. Current from test signals synthesizer is supplied to the PSh and the DUT sequential1y. The DUT output frequency, which is proportional to the measured power, is transferred to the measured power.

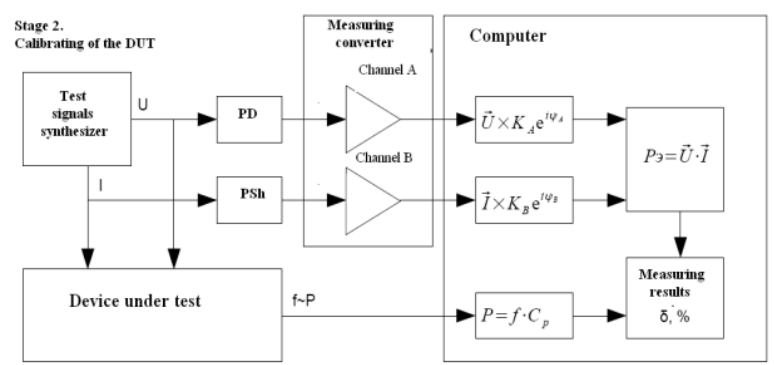

Figure 3. Calibrating of the DUT

The reference power is calculated using (5):

$$
\text { Pref }=\vec{U} \cdot \vec{I}=U_{A} \cdot I_{B} \cdot \cos \left(\varphi_{B}-\varphi_{A}\right)
$$

and then calculated the relative error of the DUT and the uncertainty of type A.

Uncertainty type B of power measurement:

$$
u_{\text {BrelP }}=\sqrt{u_{S r e l U}^{2}+u_{S r e l l}^{2}+\operatorname{tg}^{2}\left(\varphi_{B}-\varphi_{A}\right) \cdot\left(u_{S \varphi_{B}}^{2}+u_{S \varphi}^{2}\right.}
$$

where $\mathrm{u}_{\text {SrelU }}$ is relative standard uncertainty of coefficient transfer module calibration of voltage channel;

$\mathrm{u}_{\text {SrelI }}$ is relative standard uncertainty of coefficient transfer module calibration of current channel;

$\mathrm{u}_{\mathrm{S} \varphi \mathrm{A}}$ is absolute standard uncertainty of transfer coefficient phase calibration of voltage channel;

$\mathrm{u}_{\mathrm{S} \varphi \mathrm{A}}$ is absolute standard uncertainty of transfer coefficient phase calibration of current channel;

Extended relative uncertainty of calibration:

$$
U_{r e l}=2 \sqrt{u_{\text {ArelP }}^{2}+u_{\text {BrelP }}^{2}+u_{\text {relF }}^{2}},
$$

where $u_{\text {SrelF }}$ is relative standard uncertainty of frequency measurement.

As an example, the calibration table of the two reference meters with accuracy class of 0.02 is shown, which indicates relative deviation of power measurement result $\delta$ and expanded uncertainty $\mathrm{U}_{\text {rel }}$.

Table 1. The results of the reference meters calibration

\begin{tabular}{|c|c|c|c|c|c|}
\hline \multicolumn{3}{|c|}{ Mode } & \multicolumn{2}{c|}{$\delta, \%$} & \multirow{2}{*}{$U_{\text {rel, } \%}$} \\
\cline { 1 - 4 } U, V & I, A & $\cos (\varphi)$ & Meter 1 & Meter 2 & \\
\hline \multirow{2}{*}{220} & \multirow{3}{*}{1} & 1 & $-0,0046$ & 0,0050 & 0,021 \\
\cline { 3 - 6 } & & $0,5 \mathrm{~L}$ & $-0,0003$ & 0,0037 & 0,023 \\
\cline { 3 - 6 } & & $0,8 \mathrm{C}$ & $-0,0092$ & 0,0126 & 0,021 \\
\cline { 3 - 6 } & \multirow{2}{*}{5} & 1 & $-0,0010$ & 0,0079 & 0,021 \\
\cline { 3 - 6 } & & $0,5 \mathrm{~L}$ & 0,0092 & 0,0088 & 0,023 \\
\cline { 3 - 6 } & & $0,5 \mathrm{C}$ & $-0,0066$ & 0,0171 & 0,023 \\
\hline 60 & 0,05 & 1 & $-0,0063$ & 0,0032 & 0,021 \\
\hline
\end{tabular}

It should be noted that the uncertainty of coefficient transfer module calibration of channel current contributes the major part to the uncertainty of the power meters calibration.

The consequence of the use of vector measurement 
principle is that a current vector must be multiplied by the rotation operator of 90 degrees to obtain reactive power:

$$
\vec{I}_{P}=\vec{I} \times 1 \mathrm{e}^{i 90}
$$

Then the reactive power is defined as

$$
\text { Qref }=\vec{U} \cdot \vec{I}_{P}=U_{A} \cdot I_{B} \cdot \cos \left(\varphi_{B}+90-\varphi_{A}\right)
$$

Conclusions:

1. Set for power calibration of measuring instruments is created. It based on the vector measurements and has metrological traceability to AC voltage and current standards.

2. Uncertainty of measurement during calibration of power measurement means is estimated to be about $0.02 \%$

\section{References}

[1] DSTU 4116-2002 "State hierarchical scheme for instrument of measuring electrical power and power factor in the frequency range from 40 to $20,000 \mathrm{~Hz}$

[2] The Wide World of Wide-area Measurement / Phadke AG, RM de Moraes [et al.] // Power and Energy Magazine, IEEE, September-October 2008. — Vol. 6. - No. 5. - P. 52-65

[3] Mathematical modeling channel of voltage vectors measurement in control systems of electric power facilities. I.V. Yakovleva, PhD. Sc. Science; E.M Tankevych, Dr. Sc. Science; G.M Varskyy, PhD. Sc. Science. / ISSN 1997-9266. Bulletin of the Vinnytsia Polytechnic Institute. 2012, №1, p.58-61. 